\title{
Review
}

\section{Mental health policy: protecting community mental health during the COVID-19 pandemic}

\author{
Retno Lestari, ${ }^{1}$ Febri Endra Budi Setyawan² \\ ${ }^{1}$ School of Nursing, Faculty of Medicine, Universitas Brawijaya, Malang; ${ }^{2}$ Faculty of Medicine, Universitas \\ Muhammadiyah Malang, Malang, Indonesia
}

\begin{abstract}
Despite the growing interest in the treatment and management of COVID-19, communities still end up experiencing multiple stresses with mental health crisis, due to the pandemic. When this is not addressed, it causes stress in the long run, with further mental health damage in individuals and the communities. Furthermore, relevant policy related to the community mental health was identified as awareness in promoting mental issues, yet there are still failures recorded in the aspect of adequately addressing the well-being concerns. This study aims to support community mental health during the crisis, through useful information from relevant articles. It also explains some perspectives from literature reviews, case reports, with society responses from Google Scholar, Science Direct, and Proquest databases concerning recent community mental health issues, and government policy on the impact of the COVID-19 pandemic. The inclusion criteria required articles that were peer-reviewed, by using i) English language March-September 2020; ii) Presented empirical studies related to COVID-19 (quantitative and qualitative); iii) Searched terms related to the general and community mental health, their policy, and COVID-19 pandemic. This study showed 19 articles related to community mental health issues or psychological wellbeing topics. The interventions provided to strengthen community mental health during the pandemic included emergency psychological crisis treatment, hotline assistance, online counseling service with mental care course, and outpatient consultation. Therefore, community mental health issues should be addressed with physical wellness. In addition, the community plays a key role in influencing stakeholders and governments, to increase the priority in supporting mental health.
\end{abstract}

\section{Introduction}

As the effects of the COVID-19 pandemic continues, current response strategies need to emphasize more on the importance of tackling mental health issues. Despite the growing interests in the treatment and management of COVID-19, communities still experience multiple stresses, with mental health crisis due to the pandemic. When this is not addressed, it causes more stress level, feeling of overwhelmed, and further increase in mental health damage among individuals and the communities. Advanced mental health care infrastructures and well-being concern systems are needed, to provide the best appropriate treatment and care.

Generally, the government help in implementing a number of COVID-19 preventive responses, to reduce the risk of disease transmissions by hands washing, social distancing measures, staying indoors, and promoting work from home. ${ }^{1,2}$ The most important aspect in this policy, is the certain considerations for emergency conditions. However, these responses made quite a number of people feel isolated, inconvenient while working from home, and lonely. Previous research showed that, seven out of ten working staffs experienced stress during this pandemic, which in particular, is well indicated by an increase in medical prescriptions, such as, antidepressants, antianxiety, and anti-insomnia drugs. ${ }^{3}$ Long term isolation certainly affects the health of people, both physically and mentally.

Earlier studies showed varieties of mental health issues during the COVID-19 pandemic, ranging from feeling overwhelmingly stressed, grief, mistrust towards medical professionals or treatment, panic attacks, anxiety, depression, anger, fear, stigmatization, child maltreatment, abuse, suicidal ideation, etc. ${ }^{4-6}$ The health professionals and young people are the most vulnerable population, to experience mental health issues. Higher prevalence of anxiety and depressive symptoms are found among people less than 35 years old, health professionals that spent more than 3 hours/day during the pandemic, have poor sleep quality in comparison with other occupations. ${ }^{7}$ In another study, poor knowledge, lack of training, and inadequacy of personal protective equipment lead to severe distress among health professionals,, 89 with this condition weakening their immune systems, as studies found higher infection rates in practitioners' well-being. ${ }^{10}$

Furthermore, in developing mental health policy, it is very important to consider all psychological well-being issues arising in the community, including, stigmatization discrimination

Significance for public health

During the COVID-19 pandemic, communities are faced with multiple stresses, and mental health crisis. Advanced mental well-being care infrastructures, with other systems experienced a great challenge in providing the best treatment and care, due to inadequate funding. Facilitating emotional support is very important, in order to boost mental health of individuals, children, elderly, medical workers, and the community at large, during the pandemic. Also, communities need to increase mental health awareness, and prevention of its respective illnesses. Therefore, relevant articles have highlighted the importance of public health education, about adapting, and responding to the COVID-19 pandemic. Furthermore, this study help describes community mental health strategies during the crisis. 
towards COVID-19 patients and health professionals. ${ }^{11}$ Most importantly, adequate support from the entire communities, stakeholders, decision-makers, and the government is highly needed. Stigma is detrimental not just to COVID-19 patients, and also to the whole society. The negative stigma surrounding the patients, causes reluctance to attend screening tests and seek treatment, therefore, leading to speedy transmissions of the infectious disease. The World's Health Organization (WHO) provided recommendations and guidelines on how to combat stigmatization as regards COVID-19, all in a bid to help deal with mental health issues during the pandemic. ${ }^{12}$ Moreover, this was an excellent idea to help address community mental health issues, to which had many advantages for the entire communities, families, health professionals, stake holders, and policy makers. This study aims to support community mental health during the crisis, through useful information from relevant articles. Therefore, it is expected that the communities follow government's advice, comply with health policies, practice healthy lifestyles, and provide support during the crisis.

\section{Design and Methods}

This study explained some perspectives from the literature reviews, case reports, with society responses from Google Scholar, Science Direct, and ProQuest databases with regards to the recent community mental health issues, due to the COVID-19 pandemic impact. The inclusion criteria required articles that were peer reviewed, by using i) English language between March 2020 to September 2020; ii) presented empirical studies related to COVID19 (quantitative and qualitative); iii) searched terms related to psychological wellness, community mental health, COVID-19, pandemic, and mental health policy. This study showed 97 articles related to mental health issues or psychological wellness topics to be reviewed during the pandemic. Articles were then included, when they directly described community mental health issues, and its policy during outbreak or crisis or pandemic. Articles of about 19 were found eligible to be reviewed (Figure 1). Also, reasons for writing the article along with opinions about the topic were fully expressed.

\section{Results and Discussions}

Table 1 described the recommendations for strengthening mental health services. Any relevant articles, presenting evidence on mental health approaches during pandemic were also included in the analysis. Furthermore, this study was complemented by grey literature reviews from newspapers' reports, government websites while upholding the rules and regulations, with current situation updates on COVID-19.

In order to strengthen mental health policy during the pandem-
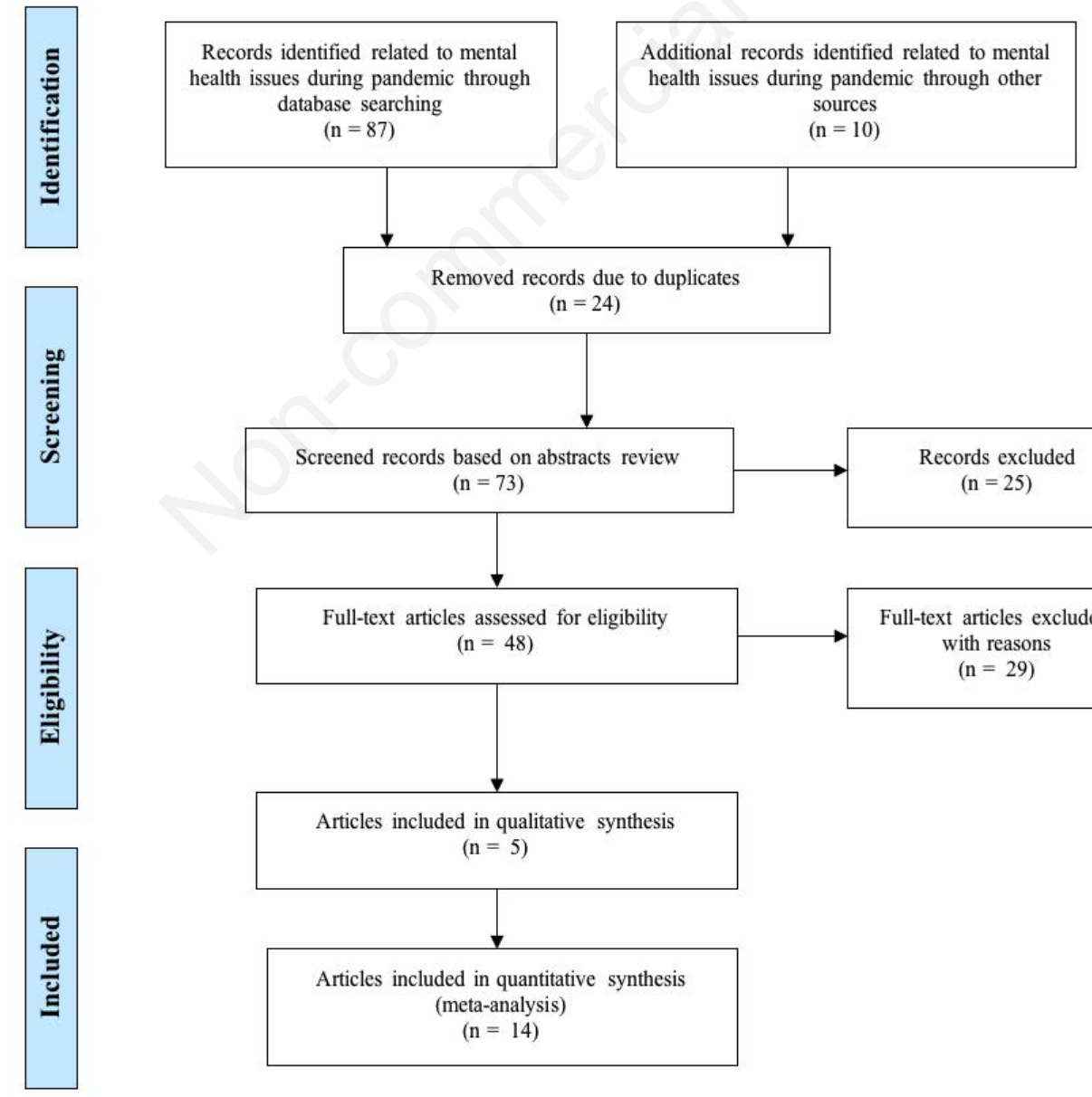

Figure 1. Flowchart of study protocol. 
ic, it is important to first identify mental health issues related to the present situation and place it in the overall process recommendations (Table 1). An interesting study in China investigated the effect of excessive exposure to social media, with about $83.1 \%$ respondents experiencing this, which in turn, influenced their mental health issues. About 48.3\% respondents had depression, 22.6\% felt anxiety, and $19.4 \%$ possessed the combination of both, respectively. ${ }^{13}$ One third of the U.S populations described mental health and financial issues related to the impact of pandemic, such as stress, anxiety, grief, and major hardships to overcome the challenges. ${ }^{14}$ Participants were reported having a moderate level of fear of having COVID-19 (M=2.84, S =1.07) and worried that their closed relatives becoming ill with this disease $(\mathrm{M}=3.53$, $\mathrm{SD}=1.03) .{ }^{15}$ Another study revealed that women suffered more symptoms than men, characterized by depression, anxiety, stress, and suicidal ideation. ${ }^{16}$ The implementation of lockdown policy and other social distancing create a more physical and psychological issues as some workers lose their jobs. ${ }^{17}$ These mental health issues are also commonly found in vulnerable people such as, psychiatric survivors, health professionals, children, adolescents, elder people, specific ethnic groups, homeless, community members, and people who live in rural and remote areas. ${ }^{18-20}$ Therefore, mental health interventions during the pandemic should focus on 6 vulnerable groups; i) confirmed COVID-19 patients; ii) suspected victims; iii) health professionals; iv) losed contact with confirmed wards; v) those who refused to get proper treatment; and vi) group of suspects in public areas. ${ }^{21}$

Furthermore, since spreading misleading information related to COVID-19 have the potential to cause distressful harm, several interventions should be provided such as emergency psychological crisis treatment, hotline assistance, online counseling service with mental health course, and outpatient consultation when needed. ${ }^{17,18,22}$ However, these online mental health services were not often available in low- and middle-class sections of developing countries. Most people were not too familiar with the use of technology, resulting in it being such a challenging task for the government and health professionals, in a bid to make available mental health services for the community. ${ }^{23}$ In Africa, partnerships with social media platforms were built, to provide sufficient information related to the pandemic, and reduce the fears, along with the stigma of COVID-19. ${ }^{24}$ Changes in school routines became stressful for children, as most did not see their peers, which resulted in further mental health issues creation. In UK, $83 \%$ children felt their mental health had worsened during the pandemic. ${ }^{25}$ For children that felt stuck while studying at home, they shared their thoughts and feelings through online application for mental health, hotline counseling services, or offline channels, with help from community mental health team. ${ }^{6,26}$ Another study stated that about $52.1 \%$ young people felt worried due to the impact of pandemic, hence, they received a great support from friends and families. ${ }^{27}$ However, specific attention needs to be paid to children with special demands, and other disabilities, as they face mental health risks more greater, due to lack of recommended therapies, or educational wants. Also, behavioural issues happening while special needs children stay at home during school hours, which results in parental stress, adding more tension to the children, should be well addressed.

There are 10 strategies made available, for reducing tension between parents and special need children: i) describe briefly what COVID-19 is; ii) restructure routine plans; iii) develop semi-structured activities; iv) enhance cognitive function, through simple games; v) engage in shared video arcade for both parties; vi) plan hobbies together; vii) online therapies; viii) media consultations; ix) frequent contact with school teachers; $x$ ) relaxation and break time for the kids. ${ }^{28}$ These strategies are used to help parents manage additional behavioural problems, during isolation at home. Elderly people often feel anxiety with depression, therefore counselling service, and psychological crisis interventions should be provided as well. Mental health interventions best for elderly people during the pandemic are i) strengthening the support environment for those with low educational level, divorced, widowed, living alone, sleep disorders, and history of mental diseases; ii) improve overall health issues, as they have weaker immune systems that are prone to infection; iii) facilitate wellness orientation along with psychological well-being support; and iv) refer to accurate medical professionals when needed, for further consultations. ${ }^{5}$

Health care workers often feel fatigue and depressed, also, they need adequate support from families, communities, and mental wellness interventions. ${ }^{29}$ Factors associated with increased risk in mental health issues among medical professionals are socio-demographic characteristics (young age, female), social aspects (support, stigma to COVID-19), job status, lack of sufficient training and experience. It is very important to facilitate emotional support, to boost their confidence, self-efficacy, and coping mechanisms in this pandemic, mindful of the fact that they have had to work under pressure, as many do not go home during the on-going crisis. ${ }^{30,31}$

Table 1. Recommendations for strengthening mental health services.

Target population Mental health strategies

Individual

Emergency psychological crisis treatment

Hotline and online counselling service

Online mental health course

Outpatient consultation when needed

\begin{tabular}{ll} 
Children & Online application for mental health counselling \\
& Continuity of care \\
& Mental health interventions \\
\hline Elderly & Counselling services \\
& Psychological crisis treatment \\
Health care workers & Adequate support from families, communities \\
& Mental health interventions \\
\hline Community & Health education about adapting and responding to COVID-19 \\
& Healthy activities during self-isolation \\
& Access to resources and healthcare services \\
& Community empowerment \\
\hline
\end{tabular}


Positive coping styles were reported to increase mental health status among individuals, such as using positive reframing, acceptance, and humour. ${ }^{16}$ Therefore, to improve community mental health during COVID-19 pandemic, medical professionals and communities need to collaborate with the government, to deliver mental health education, about adapting and responding to COVID-19, creating therapeutic environment during the pandemic, along with promoting healthy activities during self-isolation. ${ }^{31-}$ 33 It is also very important to disseminate potential access to resources and healthcare services, while also empowering communities to fight COVID-19. ${ }^{4}$

\section{Conclusions}

In conclusion, it is important that mental health issues should be addressed as well as physical well-being. Also, there is need to strengthen mental health policy to deal with psychological issues, stigma, and discrimination during the pandemic. The community plays a key role in influencing stakeholders and governments, to increase the priority in supporting mental health during COVID-19 pandemic. Therefore, this study recommends that the policy makers should develop a better understanding of community mental health needs during the pandemic and enable intermediaries to contribute for a better procedure.

Correspondence: Retno Lestari, School of Nursing, Faculty of Medicine, Universitas Brawijaya, Jl. Puncak Dieng, Kunci, Kalisongo, Kec. Dau, Malang, East Java 65151, Indonesia.

Tel. +62.341569117 - Fax: +62.341564755 .

E-mail: retno.lestari.fk@ub.ac.id

Key words: Mental health policy; community mental health; pandemic.

Acknowledgments: We are grateful to our colleagues from School of Nursing, Faculty of Medicine, Universitas Brawijaya, Malang, Indonesia, who provided insight and expertise that greatly assisted the success of this study.

Contributions: The authors contributed equally from the conception, design, providing the study materials, and writing the manuscript.

Conflict of interests: The author declares no potential conflict of interest.

Funding: This study was financially supported by School of Nursing, Faculty of Medicine, Universitas Brawijaya.

Ethics approval: Not applicable.

Conference presentation: Part of this study was presented at the $1^{\text {st }}$ International Nursing and Health Sciences Symposium, November $13^{\text {th }}$ to $15^{\text {th }} 2020$, Brawijaya University, Malang, Indonesia.

Received for publication: 14 January 2021.

Accepted for publication: 18 March 2021.

oCopyright: the Author(s), 2021

Licensee PAGEPress, Italy

Journal of Public Health Research 2021;10:2231

doi:10.4081/jphr.2021.2231

This work is licensed under a Creative Commons Attribution NonCommercial 4.0 License (CC BY-NC 4.0).

\section{References}

1. Nurhalimah N. [Upaya Bela Negara Melalui Sosial Distancing dan Lockdown untuk Mengatasi Wabah COVID-19 (Efforts to defend the country through social distancing and lockdown to overcome the COVID-19 plague)]. SSRN 2020. Accessed: 2020 April 26. Available from: https://ssrn.com/abstract= 3576405

2. Hiremath P, Suhas Kowshik CS, Manjunath M, Shettar M. COVID 19: Impact of lock-down on mental health and tips to overcome. Asian J Psychiatr 2020;51:102088.

3. Gavidia M. How has COVID-19 affected mental health, severity of stress among employees? AJMC 2020 Accessed: 2020 April 26. Available from: https://www.ajmc.com/newsroom/ how-has-COVID19-affected-mental-health-severity-of-stressamong-employees

4. Mukhtar S. Mental health and psychosocial aspects of coronavirus outbreak in Pakistan: Psychological intervention for public mental health crisis. Asian J Psychiatr 2020;51: 102069289.

5. Meng $\mathrm{H}, \mathrm{Xu} \mathrm{Y}$, Dai J, et al. The psychological effect of COVID-19 on the elderly in China. Psychiatry Res 2020;289:112983.

6. Golberstein E, Wen H, Miller BF. Coronavirus Disease 2019 (COVID-19) and mental health for children and adolescents. JAMA Pediatr 2020;174:819-20.

7. Pan Y, Xin M, Zhang C, et al. Associations of mental health and personal preventive measure compliance with exposure to COVID-19 information during work resumption following the COVID-19 outbreak in China: Cross-sectional survey study. J Med Internet Res 2020;22:e22596.

8. Joob B, Wiwanitkit V. Medical personnel, COVID-19 and emotional impact. Psychiatry Res 2020;288:112952.

9. Xiang Y-T, Yang Y, Li W, et al. Timely mental health care for the 2019 novel coronavirus outbreak is urgently needed. Lancet Psychiatry 2020;7:228-9.

10. Neto MLR, Almeida HG, Esmeraldo JD, et al. When health professionals look death in the eye: the mental health of professionals who deal daily with the 2019 coronavirus outbreak. Psychiatry Res 2020;288:112972.

11. Torales J, O'Higgins M, Castaldelli-Maia JM, et al. The outbreak of COVID-19 coronavirus and its impact on global mental health. Int J Soc Psychiatry 2020;66:317-20.

12. World Health Organization. Mental health and psychosocial considerations during the COVID-19 outbreak. Geneva: WHO; 2020.

13. Gao J, Zheng P, Jia Y, et al. Mental health problems and social media exposure during COVID-19 outbreak. PLoS One 2020;15:e0231924.

14. Tanne JH. Covid-19: Mental health and economic problems are worse in US than in other rich nations. BMJ 2020;370:m3110.

15. Newby JM, O'Moore K, Tang S, et al. Acute mental health responses during the COVID-19 pandemic in Australia. PLoS One 2020;15:e0236562.

16. Gurvich C, Thomas N, Thomas EH, et al. Coping styles and mental health in response to societal changes during the COVID-19 pandemic. Int J Soc Psychiatry 2020;20764 020961790

17. Mumtaz M. COVID-19 and mental health challenges in Pakistan. Int J Soc Psychiatry 2020.

18. Pedrosa AL, Bitencourt L, Fróes ACF, et al. Emotional, behavioral, and psychological impact of the COVID-19 pandemic. Front Psycho. 2020;11:566212. 
19. Otu A, Charles CH, Yaya S. Mental health and psychosocial well-being during the COVID-19 pandemic: the invisible elephant in the room. Int J Ment Health Syst 2020;14:38.

20. Pan K-Y, Kok AAL, Eikelenboom M, et al. The mental health impact of the COVID-19 pandemic on people with and without depressive, anxiety, or obsessive-compulsive disorders: a longitudinal study of three Dutch case-control cohorts. Lancet Psychiatry 2021;8:P121-9.

21. Dong L, Bouey J. Public mental health crisis during COVID19 pandemic, China. Emerg Infect Dis 2020;26:1616-8.

22. Yao H, Chen J-H, Xu Y-F. Rethinking online mental health services in China during the COVID-19 epidemic. Asian J Psychiatr 2020;50:102015.

23. Hopman J, Allegranzi B, Mehtar S. Managing COVID-19 in low- and middle-income countries. JAMA 2020;323:1549-50.

24. Lee J. Mental health effects of school closures during COVID19. Lancet Child Adolesc Health 2020;4:421.

25. Xie X, Xue Q, Zhou Y, et al. mental health status among children in home confinement during the Coronavirus Disease 2019 outbreak in Hubei Province, China. JAMA Pediatr 2020;174:898-900.

26. Narzisi A. Handle the autism spectrum condition during Coronavirus (COVID-19) stay at home period: Ten tips for helping parents and caregivers of young children. Brain Sci
2020;10:207.

27. Zhang Y, Ma ZF. Impact of the COVID-19 pandemic on mental health and quality of life among local residents in Liaoning Province, China: A cross-sectional study. Int J Environ Res Public Health 2020;17:2381.

28. Du J, Dong L, Wang T, et al. Psychological symptoms among frontline healthcare workers during COVID-19 outbreak in Wuhan. Gen Hosp Psychiatry 2020;67:144-5.

29. Ricci-Cabello I, Meneses-Echavez JF, Serrano-Ripoll MJ, et al. Impact of viral epidemic outbreaks on mental health of healthcare workers: A rapid systematic review. medRxiv 2020.04.02.20048892.

30. Sun N, Shi S, Jiao D, et al. A qualitative study on the psychological experience of caregivers of COVID-19 patients. Am J Infecti Control 2020;4:20048892.

31. Ju Y, Zhang Y, Wang X, et al. China's mental health support in response to COVID-19: progression, challenges and reflection. Global Health 2020;16:102.

32. Dotson S, Ciarocco S, Koh KA. Disaster psychiatry and homelessness: creating a mental health COVID-19 response. Lancet Psychiatry 202;7:1006-8.

33. Li W, Yang Y, Liu ZH, et al. Progression of mental health services during the COVID-19 outbreak in China. Int J Biol Sci 2020;16:1732-8. 